statistically significant change in syphilis testing rate among males $(\mathrm{p}=0.03)$.

Conclusion Our campaign appeared to have reached people living in BC. Syphilis knowledge was high in our pre-campaign sample, which may represent a more educated cohort. Nevertheless, those who saw the campaign scored higher. Syphilis testing rate among males increased significantly after campaign implementation. Work is underway to evaluate the campaign among gbMSM.

Disclosure No significant relationships.

\section{P758 USE OF A RAPID SYPHILIS TEST FOR SYPHILIS DIAGNOSIS AMONG PATIENTS PRESENTING TO AN STI CLINIC IN NORTH CAROLINA, USA}

${ }^{1}$ Arlene Seña*, ${ }^{2}$ Katie Mallette. ' University of North Carolina at Chapel Hill, Division of Infectious Diseases, Chapel Hill, USA; ${ }^{2}$ Durham County Department of Public Health, Durham, USA

\subsection{6/sextrans-2019-sti.816}

Background Rapid treponemal antibody-based tests have been utilized for syphilis screening and diagnosis. In the United States (US), the Syphilis Health Check (SHC, Trinity Biotech) is the only FDA-cleared rapid test. However, there is a high proportion of false positives with SHC screening. We evaluated the performance of the SHC for syphilis diagnosis among patients presenting to an STI clinic in Durham, North Carolina (NC), US.

Methods Beginning in September 2017, the SHC was routinely performed for any patient in the STI clinic for whom a stat rapid plasma reagin (RPR) was requested and had no history of prior syphilis. The SHC and RPR were performed at the on-site laboratory using venous blood specimens. Confirmatory treponemal tests (e.g. enzyme immunoassay) were conducted for specimens with discrepant results at the state public health laboratory. We determined the performance of the SHC results in comparison with either a combined positive RPR/treponemal test or positive treponemal test as the standard for a "true positive."

Results Over a 16-month period, 182 unique patients who presented with symptoms and/or as contacts to syphilis underwent testing with a stat RPR and the SHC; the majority were African-American (83\%) and male (74\%). The SHC was positive in 66 patients; 9 of these cases had nonreactive RPRs but positive treponemal tests. The estimated sensitivity of the SHC was $91.3 \%(63 / 69)$, with a specificity of $97.3 \%(110 / 113)$. Of the 3 specimens with positive SHC but negative confirmatory testing, one was from an asymptomatic patient with contact to syphilis and an RPR 1:1.

Conclusion Rapid syphilis testing can be used to assist in the immediate diagnosis of STI clinic patients with suspected syphilis based on symptoms or exposure. The SHC had an acceptable sensitivity and specificity, with a low proportion of falsepositives among a population with a high likelihood of syphilis infection.

Disclosure No significant relationships.

\section{P759 \\ EVALUATION OF THE FULLY AUTOMATED BIO-RAD BIOPLEX 2200 SYPHILIS TOTAL \& RPR ANTIBODY DETECTION ASSAY}

${ }^{1}$ Vincent Boissonneault ${ }^{*},{ }^{1}$ Valérie Martel-Laferrière, ${ }^{2}$ Bouchra Serhir, ${ }^{1}$ Claude Fortin. ${ }^{1}$ Centre Hospitalier de I'Université de Montréal, Departement De Microbiologie Infectiologie, Montreal, Canada; ${ }^{2}$ Institut National de Santé Publique du Québec, Montréal, Canada

\subsection{6/sextrans-2019-sti.817}

Background Laboratory diagnostic of syphilis is based on both treponemal and non-treponemal assays. While automated treponemal assays are available, non-treponemal antibody testing continue to require extensive hands on time. The BioPlex 2200 Syphilis Total \& RPR assay by Bio-Rad Laboratories is a fully automated assay that detects both treponemal and nontreponemal antibodies with on board RPR titer capability.

Methods We compared the BioPlex Total \& RPR assay with the BiopPex IgG assay and with the Wampole Impact RPR card test for the treponemal and non-treponemal antibodies detection respectively. A total of 504 prospective sera sent to our laboratory for syphilis testing were tested with both BioPlex assays to establish agreement of treponemal results. Also, a panel of 52 sera with known titer values was provided by our reference laboratory. These sera were tested with the BioPlex Total \& RPR assay and the manual Wampole RPR card to compare titer results, using essential agreement within 1 dilution range and correlation of $\log 2$ transformed titer values with Spearman coefficient.

Results Of the 504 sera tested, 112 were positive for treponemal antibodies with BioPlex Total \& RPR and 109 with BioPlex IgG. Positive, negative and overall agreements were 95\% (104/109)(95\% CI [89\%, 98\%]), 98\% $\quad(387 / 395)(95 \% \quad$ CI [96\%, 99\%]) and 97\% (491/504)(95\% CI [95\%, 99\%]) respectively for these sera. Essential agreement within 1 dilution of quantitative RPR titer results for the 52 sera panel was $90 \%$ and the Spearman correlation coefficient of titers obtained with the two assays was 0.926 with a $\mathrm{p}<0.001$.

Conclusion The BioPlex 2200 Syphilis Total \& RPR assay is a fully automated assay that compares favorably with the BioPlex 2200 Syphilis IgG assay and that yields non treponemal titers that correlates favorably with a traditional manual RPR card test. Reliable automated non treponemal assays are needed.

Disclosure No significant relationships.

\section{P760 SENSITIVITY AND SPECIFICITY OF THE QUANTITATIVE TEST FOR TPPA AND RPR FOR DIAGNOSIS OF CONGENITAL SYPHILIS IN NEONATES}

${ }^{1}$ Xiangdong Gong*, ${ }^{2}$ Minzhi Wu, ${ }^{1}$ Liang Zhao. ${ }^{1}$ Institute of Dermatology, Chinese Academy of Medical Sciences and Peking Union Medical College, STD Clinic, Nanjing, China; ${ }^{2}$ The Fifth People's Hospital of Suzhou, Suzhou, China

\subsection{6/sextrans-2019-sti.818}

Background Diagnosis of congenital syphilis in neonates is difficult as TP-specific IgG can be passively transferred from mother to newborn, a reactive serologic test at birth does not 